\title{
Obesity and obesity-related comorbidities in a Canadian First Nation population*
}

\author{
S. G. Bruce, PhD (1); N. D. Riediger, MSc (1); J. M. Zacharias, MD, MSc (1); T. K. Young, MD, PhD (2)
}

\begin{abstract}
* This article is part of a joint publication initiative between Chronic Diseases in Canada and Preventing Chronic Disease. Chronic Diseases in Canada is the secondary publisher, while Preventing Chronic Disease is the primary publisher. The primary publication can be found at Bruce SG, Riediger ND, Zacharias JM, Young TK. Obesity and obesity-related comorbidities in a Canadian First Nation population. Prev Chronic Dis 2011;8(1). http://www.cdc.gov/pcd/issues/2011/jan/09_0212.htm
\end{abstract}

\section{Abstract}

Introduction: Rates of obesity are higher among Canada's Aboriginal First Nations populations than among non-First Nations populations. We studied obesity and obesityrelated illness in a Manitoba First Nation community.

Methods: We conducted a screening study of diabetes and diabetes complications in 2003, from which we drew a representative sample of Manitoba First Nation adults $(\mathrm{N}=483)$. We assessed chronic disease and chronic disease risk factors.

Results: Prevalence of obesity and associated comorbidities was higher among women than men. By using multivariate analysis, we found that factors significantly associated with obesity among women were diastolic blood pressure, insulin resistance, and employment status. Among men, factors were age, apolipoprotein A1 level, apolipoprotein B level, and insulin resistance. Seventy-five percent of study participants had at least 1 of the following conditions: obesity, dyslipidemia, hypertension, or diabetes. Comorbidity was high even among the youngest age groups; $22 \%$ of men and $43 \%$ of women aged 18 to 29 had 2 or more chronic conditions. Twenty-two percent of participants had undiagnosed hypertension. Participants with undiagnosed hypertension had significantly more chronic conditions and were more likely to have microalbuminuria than were those without hypertension. The number of chronic conditions was not significantly different for participants with newly diagnosed hypertension than for those with previously diagnosed hypertension.

Conclusions: The prevalence of obesity and other chronic conditions in the study community is high, especially considering the number of young people. Community-based interventions are being undertaken to reduce the excessive rate of illness.

Keywords: cardiovascular, community health, diabetes, minority, obesity, screening, Type 2 diabetes, First Nations, North American Indians

\section{Introduction}

The Canadian First Nations population has poorer overall health than does the general Canadian population, ${ }^{1}$ specifically in terms of chronic diseases, chronic disease risk factors, ${ }^{2}$ and injuries and accidents. ${ }^{3}$ In
Canada, First Nations peoples are 1 of 3 constitutionally recognized Aboriginal groups; the other 2 are the Métis and the Inuit. In this article, we use the term Aboriginal to report on research that included 2 or more of these distinct groups if no distinction was made between the groups in the analysis. However, if the research included only 1 group, we have identified that group. According to the 2005-2006 Canadian Community Health Survey, the prevalence of obesity among people who self-identified as Aboriginal and who did not live on reserve land was $20 \%$ in Canada's north (Yukon, Northwest Territories, and Nunavut) and $23 \%$ in the rest of Canada. ${ }^{4}$

Obesity prevalence appears to be higher among First Nations peoples living on reserves. In Sandy Lake, Ontario, the prevalence of obesity (body mass index [BMI] $\geq 30 \mathrm{~kg} / \mathrm{m}^{2}$ ) was $50 \%$ for men and $65 \%$ for women. ${ }^{5}$ Furthermore, in a First Nation community in Quebec, 91\% of study participants from a sample of 172 were abdominally obese. ${ }^{6}$ The prevalence of obesity was $55 \%$ among a sample of Alberta First Nation people and 49\% among a sample of Métis people.?

Prevalence of obesity-related comorbidities is also high among Canadian First Nations people. The prevalence of diabetes among Canadian First Nations populations is 3 to 5 times higher than among the general Canadian population. ${ }^{5-8}$ Hypertension, dyslipidemia, metabolic syndrome, and diabetes complications such as cardiovascular disease (CVD), stroke, retinopathy, neuropathy, and nephropathy are also major contributors to poor health. ${ }^{2,5-10} \mathrm{CVD}$ is the leading cause of death in Canada, and Aboriginal populations have twice the CVD death rate of non-Aboriginal populations. $^{2}$ In a random sample, the rate of CVD was $18 \%$ among Canadian Aboriginal people and $8 \%$ among people of European ancestry. $^{2}$ 
Despite the evidence of excess obesity, diabetes, and related metabolic conditions among Canada's First Nations populations, few researchers have investigated their coexistence in this population. Our purpose was to explore the magnitude and effect of obesity and obesity-related comorbidities in a Manitoba First Nation.

\section{Methods}

Our methods have been previously described. ${ }^{10}$ Briefly, 483 eligible residents of a Manitoba First Nation community volunteered in 2003 to participate in a screening study for diabetes and diabetes complications. A total of 1356 eligible participants included nonpregnant adults aged 18 years or older who were Registered Indians and who were residents of the community. Our sample (36\%, 483 of 1356) is representative of eligible participants by age and sex. ${ }^{10} \mathrm{~A}$ registered nurse drew venous samples to measure glucose, hemoglobin A1c, insulin, total cholesterol, high-density lipoprotein (HDL) cholesterol, triglycerides, apolipoprotein A1 (apoA1), total apolipoprotein B (apoB), and homocysteine levels from fasting participants (low-density lipoprotein [LDL] cholesterol was calculated).

A registered nurse or trained research assistant administered a 17-item questionnaire that included standard demographic data (age, sex, employment status, education level), current and past smoking status, number of cigarettes smoked per day, previous diagnosis of diabetes and hypertension ("Have you ever been told by a doctor that you have diabetes? How long have you had diabetes?"), and current medication use. Standard techniques were used to obtain anthropometric measures. ${ }^{11}$ Height was measured via metric wall tape and set square to the nearest $0.5 \mathrm{~cm}$; weight was measured on a balance scale to the nearest $0.1 \mathrm{~kg}$; waist circumference was measured at noticeable waist narrowing or at the level of the 12th rib, to the nearest $0.5 \mathrm{~cm}$; and hip circumference was measured at the level of the symphysis pubis and the largest area of the buttocks to the nearest $0.5 \mathrm{~cm} .{ }^{11}$
Abdominal obesity was defined as waist circumference greater than $102 \mathrm{~cm}$ for men and greater than $88 \mathrm{~cm}$ for women. ${ }^{10}$ Diabetes was defined as a fasting plasma glucose of $7.0 \mathrm{mmol} / \mathrm{L}$ or higher, or a previous diagnosis; impaired fasting glucose was defined as a fasting plasma glucose of 6.1 to $6.9 \mathrm{mmol} / \mathrm{L}^{12}$ Hypertension was defined as systolic blood pressure higher than $140 \mathrm{mmHg}$ or diastolic blood pressure higher than $90 \mathrm{mmHg}$, or a previous diagnosis. Dyslipidemia was defined as a plasma triglyceride level of $1.7 \mathrm{mmol} / \mathrm{L}$ or higher and HDL cholesterol level of 1.03 $\mathrm{mmol} / \mathrm{L}$ or less for men or $1.30 \mathrm{mmol} / \mathrm{L}$ or less for women. Metabolic syndrome was defined using Adult Treatment Panel III criteria. ${ }^{13}$ Insulin resistance was estimated through the homeostasic model assessment (HOMA), which is calculated as follows: [(insulin [pmol] x 0.139) x (glucose [mmol/L]/22.5)]. Microalbuminuria was defined as an albumin-to-creatinine ratio higher than $2.0 \mathrm{mg} / \mathrm{mmol}$ for men and higher than $2.8 \mathrm{mg} / \mathrm{mmol}$ for women. Neuropathy was defined as presence of numbness, tingling, pain, and loss of protective sensation determined through application of the 10-g Semmes-Weinstein monofilament wire system (Sensory Testing Systems, Baton Rouge, Louisiana). ${ }^{14}$ A registered nurse completed the foot examination and applied the 10-g monofilament. The University of Manitoba Health Research Ethics Board approved the project.

Statistical analyses were completed by using SPSS version 16 for Windows (IBM, Chicago, Illinois). We used $\chi^{2}$ tests to detect differences between the sexes for chronic disease prevalence, risk factors, and sociodemographic variables. We compared differences between the sexes on variables that were continuously distributed by using $t$ tests or Mann-Whitney tests for variables with a nonnormal distribution. Differences in the number of chronic health conditions by age group and sex and number of comorbidities by hypertensive status were determined by using $\chi^{2}$ tests. Tests were 2 -tailed and differences were considered significant at $p<.05$. We used logistic regression to estimate odds ratios (ORs) for obesity and microalbuminuria with $95 \%$ confidence intervals (CIs). Participants with missing values were excluded from analyses. No pattern was found for missing values by sex, age group, chronic disease, or risk factor variables.

\section{Results}

The demographic and health status characteristics of the study sample describe a young population with low education and high unemployment (Table 1). The prevalence of smoking, diabetes, hypertension, and overweight and obesity was high among study participants. Waist circumference was available for 259 of the 264 obese participants; $96 \%$ (250 of 259) had waist circumferences that placed them at high risk for adverse health outcomes. ${ }^{9}$ We found no significant differences between men and women in prevalence of diabetes or hypertension. However, the prevalence of dyslipidemia among women (38\%) was significantly higher than among men (26\%).

\section{Overall obesity and abdominal obesity}

We used BMI and waist circumference to classify participants as obese by age and sex (Figure 1). Almost $50 \%$ of men and $65 \%$ of women were obese as defined by BMI, and $53 \%$ of men and $81 \%$ of women had abdominal obesity. Obesity was more common among women than men according to BMI $\left(\chi^{2}=14.62, p<.001\right)$ and abdominal obesity $\left(\chi^{2}=41.38, p<.001\right)$. The prevalence of $\mathrm{BMI} \geq 30 \mathrm{~kg} / \mathrm{m}^{2}$ was higher among women aged 18 to 29 years than among men of the same age group $\left(\chi^{2}=9.06, p<\right.$ $.01)$. Abdominal obesity was significantly more common for women than for men in all age groups except 40 to 49 years. Threequarters of women aged 18 to 29 years had abdominal obesity (Figure 1). 
TABLE 1

Characteristics of First Nation population $(\mathrm{N}=483)$, Manitoba, Canada, 2003

\begin{tabular}{|c|c|}
\hline Characteristic $^{\mathrm{a}}$ & Value \\
\hline \multicolumn{2}{|l|}{ Sex, n (\%) } \\
\hline Men & $230(48)$ \\
\hline Women & $253(52)$ \\
\hline Age, $y$, mean (SD) & $37.8(12.3)$ \\
\hline \multicolumn{2}{|l|}{ Education $(n=469), n(\%)$} \\
\hline Grade 9 or higher & $220(47)$ \\
\hline Lower than grade 9 & $249(53)$ \\
\hline \multicolumn{2}{|l|}{ Employment status ( $n=476), n(\%)$} \\
\hline Employed & $137(29)$ \\
\hline Unemployed & $339(71)$ \\
\hline \multicolumn{2}{|l|}{ Ever smoked $(n=477), n(\%)$} \\
\hline Yes & $391(82)$ \\
\hline No & $86(18)$ \\
\hline \multicolumn{2}{|l|}{ Current smoker $(\mathrm{n}=471), \mathrm{n}(\%)$} \\
\hline Yes & $349(74)$ \\
\hline No & $122(26)$ \\
\hline \multicolumn{2}{|l|}{ BMI, $\mathrm{kg} / \mathrm{m}^{2}(\mathrm{n}=468), \mathrm{n}(\%)$} \\
\hline$<25.0$ & $76(16)$ \\
\hline $25.0-29.9$ & $128(27)$ \\
\hline$\geq 30.0$ & $264(56)$ \\
\hline  & $252(53)$ \\
\hline Abdominal obesityc $(n=464)$ & $313(68)$ \\
\hline Diabetes $^{\mathrm{d}}(\mathrm{n}=483), \mathrm{n}(\%)$ & $140(29)$ \\
\hline Hypertension $^{\mathrm{e}}(\mathrm{n}=472), \mathrm{n}(\%)$ & $201(43)$ \\
\hline Dyslipidemia $^{f}(\mathrm{n}=483), \mathrm{n}(\%)$ & $155(32)$ \\
\hline Microalbuminuria $8(n=466), n(\%)$ & $94(20)$ \\
\hline
\end{tabular}

Abbreviations: SD, standard deviation; BMI, body mass index.

${ }^{a}$ Numerators vary from 464 to 483 because not all participants completed the full protocol.

${ }^{\mathrm{b}}$ Defined using Adult Treatment Panel III criteria. ${ }^{13}$

c Defined as $>102 \mathrm{~cm}$ for men and $>88 \mathrm{~cm}$ for women.

${ }^{\mathrm{d}}$ Defined as a previous diagnosis or fasting blood glucose $\geq 7.0 \mathrm{mmol} / \mathrm{L}$.

${ }^{e}$ Defined as systolic blood pressure $>140 \mathrm{~mm} \mathrm{Hg}$ or diastolic blood pressure $>90 \mathrm{~mm} \mathrm{Hg}$ or previous diagnosis.

${ }^{\mathrm{f}}$ Defined as plasma triglyceride level $\geq 1.7 \mathrm{mmol} / \mathrm{L}$ and $\mathrm{HDL}$ cholesterol level $\leq 1.03 \mathrm{mmol} / \mathrm{L}$ for men $0 \mathrm{r} \leq 1.30 \mathrm{mmol} / \mathrm{L}$ for women.

${ }^{8}$ Defined as an albumin-to-creatinine ratio $>2.0 \mathrm{mg} / \mathrm{mmol}$ for men and $>2.8 \mathrm{mg} / \mathrm{mmol}$ for women.

\section{FIGURE 1}

Prevalence of obesity by age and sex in a Canadian First Nation population.

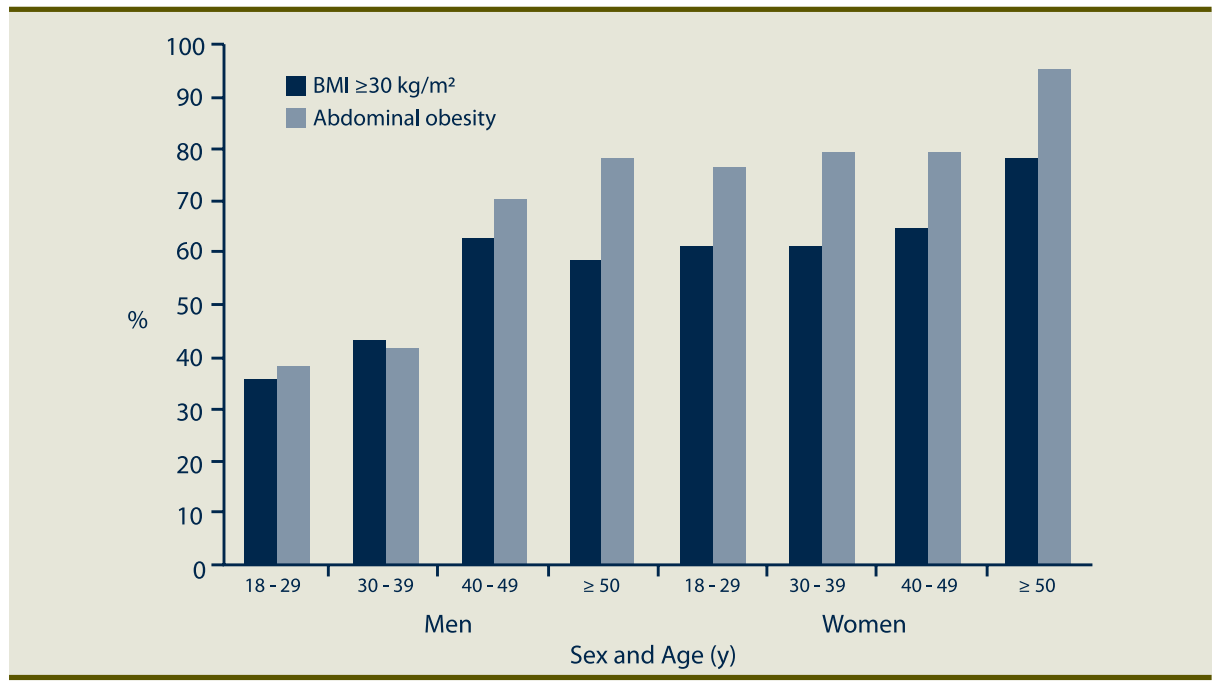

Abbreviations: BMI, body mass index.

Abdominal obesity was defined as waist circumference greater than $102 \mathrm{~cm}$ for men and greater than $88 \mathrm{~cm}$ for women.

[A tabular version of this figure is also available.]
Given the differences in obesity between men and women and the high prevalence of abdominal obesity, we determined factors associated with abdominal obesity for each sex by using multivariable backward stepwise logistic regression. Variables included in the models were those that were significantly associated with abdominal obesity in bivariate analyses. For women those variables were age; systolic and diastolic blood pressure; triglyceride, apoA1, and apoB levels; insulin resistance; education; and employment status. For men variables included in the model were age; systolic and diastolic blood pressure; triglyceride, apoA1, and apoB levels; insulin resistance; and microalbuminuria (Table 2).

For women, the odds of abdominal obesity increased with diastolic blood pressure and insulin resistance. In addition, the odds of obesity were lower for women who were employed than for those who were unemployed. Among men, abdominal obesity was associated with increasing age, insulin resistance, lower apoA1, and higher apoB levels.

\section{Comorbidities}

We determined the extent of comorbidity among this population for 4 chronic conditions: obesity, diabetes, hypertension, and dyslipidemia. The distribution of chronic conditions by age and sex (Figure 2) showed that women aged 18 to 29 and aged 50 or older had significantly more chronic conditions than men of the same age groups. Twenty-two percent (16 of 73) of men and $43 \%$ (30 of 69) of women aged 18 to 29 had 2 or more preventable chronic conditions. Among participants with abdominal obesity, $48 \%$ (147 of 303) had hypertension and $35 \%$ (111 of 313) had diabetes. Thirty-seven percent (54 of 147) of the hypertension and $26 \%$ (29 of $111)$ of diabetes cases among these participants were undiagnosed.

\section{Undiagnosed hypertension}

Overall, $22 \%$ (72 of 337) of study participants had undiagnosed hypertension. We compared the extent of comorbidity for participants with newly diagnosed hypertension and 2 groups: 1) participants who were not hypertensive and 2) 
TABLE 2

Odds of abdominal obesity by sex, First Nation population $(\mathrm{N}=483)$, Manitoba, Canada, 2003

\begin{tabular}{rlrrrrr}
\hline \multicolumn{1}{r}{ Sex } & \multicolumn{1}{c}{ Risk Factor } & \multicolumn{2}{c}{$\beta$ (SE) } & \multicolumn{2}{c}{ OR $(95 \% \mathrm{CI})$} & $p$-value ${ }^{\mathrm{a}}$ \\
\hline \multirow{3}{*}{ Women } & Currently employed & -1.16 & $(0.45)$ & 0.31 & $(0.13-0.76)$ & .01 \\
& Diastolic blood pressure & 0.05 & $(0.02)$ & 1.05 & $(1.01-1.10)$ & .03 \\
& Insulin resistance & 1.14 & $(0.205)$ & 0.31 & $(0.13-0.76)$ & .01 \\
& Age & 0.05 & $(0.01)$ & 1.05 & $(1.02-1.08)$ & .001 \\
\multirow{3}{*}{ Men } & ApoA1 & -3.06 & $(1.20)$ & 0.05 & $(0-0.49)$ & .01 \\
& ApoB & 1.54 & $(0.68)$ & 4.64 & $(1.22-17.65)$ & .02 \\
& Insulin resistance & 0.33 & $(0.08)$ & 1.40 & $(1.19-1.63)$ & $<.001$ \\
\hline
\end{tabular}

Abbreviations: SE, standard error; OR, odds ratio; $\mathrm{CI}$, confidence interval; apo, apolipoprotein.

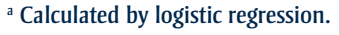

FIGURE 2

Percentage of sample with chronic conditions (obesity, diabetes, hypertension, dyslipidemia) by age and sex in a Canadian First Nation population.



[A tabular version of this figure is also available.]

participants with a previous diagnosis of hypertension (Table 3). Participants with newly diagnosed hypertension were significantly more likely to have more chronic conditions than were the normotensive participants. However, we found no significant differences in extent of comorbidity between those with newly diagnosed hypertension and those with previously diagnosed hypertension. In terms of outcomes, the adjusted odds of microalbuminuria among people with newly diagnosed hypertension were almost 2 times higher than among those without hypertension. The adjusted odds of microalbuminura among those with previously diagnosed hypertension were almost 5 times higher than among those without hypertension $[<.001$ in Table $]$.

\section{Discussion}

The prevalence of obesity in the study population is among the highest reported for a Canadian First Nation community on a reserve $e^{6,7}$ and is substantially higher than that among the general Canadian ${ }^{4}$ and offreserve Aboriginal populations. ${ }^{4,15}$ The high prevalence of obesity in the study population is concerning given the etiologic role of obesity in diabetes, heart disease, stroke, and some cancers. The prevalence of diabetes that we found is one of the highest reported among Canadian First Nations populations. ${ }^{6,7,16}$

One finding of concern is the high prevalence of obesity among young adults, especially young women of reproductive age. The relationships between maternal obesity and gestational diabetes, type 2 diabetes, poor birth outcomes, and development of obesity and type 2 diabetes among offspring are well documented. ${ }^{17-20}$ Thus, the prevalence of obesity in this young study population warrants intervention. These findings are important for 2 reasons: 1) participants developed chronic conditions at young ages, and 2) hypertension and diabetes cases were undiagnosed among a large proportion of obese participants.

Results from logistic regression confirmed established associations between obesity and plasma lipid levels, hypertension, insulin resistance, and sociodemographic factors in the study population. The sexspecific regression analyses did not include lipids as a predictive factor for abdominal obesity among women. We offer 2 possible reasons for this. First, the prevalence of abdominal obesity was high among women in all age groups but the presence of abnormal lipid levels was not. These age differences may have been blunted because our outcome (obesity) was present in all age groups. Second, previous research has shown significant sex differences in the relationship between adiposity and plasma lipids. ${ }^{21}$ Because abnormal lipid levels did occur among women, this finding warrants further examination.

We found a high prevalence of comorbidity even among the youngest age groups. The Diabetes and Related conditions in Urban Indigenous people in the Darwin region (DRUID) study also found high numbers of cardiovascular comorbidities among Australian Aborigines, and a higher number of comorbidities with increasing age. ${ }^{22}$ A large proportion of the study participants had undiagnosed diabetes and hypertension, despite the known strong correlations among obesity, diabetes, dyslipidemia, and hypertension $^{23}$ (we could not determine the extent of undiagnosed dyslipidemia among study participants because we did not ask them to self-report abnormal lipid levels). In a previous study, risk factors for not having blood pressure measured included male sex, never being married, not having a regular physician, being younger, and belonging to an Aboriginal or other ethnic minority group. ${ }^{24}$ In our study, the likelihood of not having hypertension 
Table 3

Comorbidities and risk for microalbuminuria by hypertension status, First Nation population, Manitoba, Canada, 2003

\begin{tabular}{|c|c|c|c|c|c|c|c|c|}
\hline \multirow{3}{*}{$\begin{array}{c}\text { Participants' } \\
\text { hypertension status }(\mathrm{N}=453)^{\mathrm{b}}\end{array}$} & \multicolumn{5}{|c|}{ No. of participants (\%) } & \multicolumn{3}{|c|}{ Risk for microalbuminuria ${ }^{a}$} \\
\hline & \multicolumn{4}{|c|}{ No. of comorbidities } & \multirow{2}{*}{$p$-value ${ }^{c}$} & \multirow{2}{*}{$\beta$ (SE) } & \multirow{2}{*}{ Odds Ratio } & \multirow{2}{*}{$p$-value ${ }^{c}$} \\
\hline & 0 & 1 & 2 & 3 & & & & \\
\hline No hypertension $(n=263), n(\%)$ & $111(42)$ & $88(33)$ & $50(19)$ & $14(5)$ & 1 [Reference] & 1 [Reference] & 1.000 & 1 [Reference] \\
\hline Newly diagnosed hypertension $(\mathrm{n}=72), \mathrm{n}(\%)$ & $17(24)$ & $20(28)$ & $19(26)$ & $16(22)$ & $<.001$ & $0.653(0.48-0.82)$ & 1.921 & $<.001$ \\
\hline Previously diagnosed hypertension $(n=118), n(\%)$ & $18(15)$ & $36(31)$ & $38(32)$ & $26(22)$ & .510 & $1.542(1.22-1.86)$ & 4.673 & $<.001$ \\
\hline
\end{tabular}

Abbreviation: SE, standard error.



${ }^{\mathrm{b}}$ In this analysis, we included only participants for whom values were available for all variables.

'Calculated by using $\chi 2$ test.

diagnosed was higher for men (OR, 3.27; $95 \%$ CI, 1.74-6.10; $p<.01)$ and younger participants (OR, 1.04; $95 \%$ CI, 1.01-1.07; $p<.001)$.

In our study, the undiagnosed hypertension was not benign. The extent of comorbidity among participants with newly diagnosed hypertension was similar to that for those with previously diagnosed hypertension. In addition, the risk for microalbuminuria was significantly higher among participants with newly diagnosed hypertension compared with those without hypertension but not significantly different between those with newly diagnosed hypertension and those with previously diagnosed hypertension. This suggests that newly diagnosed hypertension among participants had existed for some time. The association between hypertension and outcomes such as CVD and stroke warrants vigilant screening on the part of health care providers, especially in high-risk populations. Some participants in our "newly diagnosed" group may have been told by a physician that they did have hypertension, but they may not have remembered or they may have not understood. However, none were receiving antihypertensive treatment, so they probably had not received a hypertension diagnosis before our study.

The study is subject to limitations. First, our sample was based on volunteers and therefore may not be representative of the community as a whole or of other Canadian First Nations communities. A screening study based on a volunteer sample may attract primarily healthy people who are motivated to learn more about their health, resulting in an underestimation of illness. On the other hand, a screening study can attract people who already have health problems and are seeking additional medical assistance, which may result in an overestimation of the prevalence of illness in a population. We do not think our sample was overrepresented by either group because men and women were equally represented, and the age distribution of our sample matched that of the eligible population. ${ }^{10}$ Another indication that the prevalence of illness in the community was not overstated is that only half of the community members known to have diabetes participated in the study. None of the 15 people with end-stage renal disease participated, and only 3 of 10 community members with amputations participated. ${ }^{10}$ The prevalence of chronic disease and risk factors that we report are not substantially out of line with previous research.

A second limitation is the use of a fasting glucose test rather than a glucose tolerance test. More people with diabetes may have been identified if 2 -hr glucose tolerance tests were conducted. However, our protocol is acceptable for epidemiologic research. A third limitation is that we did not validate the self-reported hypertension or diabetes status measures with local health care providers, so we may have underestimated self-reported prevalence and therefore overestimated undiagnosed cases. However, we have previously reported lack of adherence with standards of care in this community in relation to foot examinations among people with diabetes, ${ }^{10}$ so participants may not have been tested for diabetes and hypertension even when indicated. Finally, the study is crosssectional, so we cannot infer the temporal sequence of events.
The prevalence of obesity in this population is among the highest reported among Canadian First Nations populations, particularly among women in their reproductive years. The extent of obesity-related comorbidity in this population is high even among young adults, and women at almost every age have a significantly higher rate of comorbidity than do men. A sizable proportion of participants have undiagnosed hypertension that may have been present for some time, given the significant associations with the other chronic diseases and microalbuminuria. The prevalence of cardiovascular and renal disease risk factors in this population may portend a larger prevalence of cardiovascular and renal disease. In addition, given the influence of maternal obesity and diabetes on the health of offspring, an increase in childhood obesity and type 2 diabetes could occur in the community.

An increasing prevalence of obesity and obesity-related conditions is not inevitable, however. Many prevention activities are under way. First, a research intervention in the community is focused on preventing gestational diabetes through controlling weight gain during pregnancy with exercise and diet. Second, the community operates a fitness center that has good equipment and instruction. Third, the health center offers education on diet, exercise, and wellness. Fourth, walking groups for youth and adults are organized through the health center. Fifth, activity programs for young people operate out of the local schools. However, given the wellestablished effect of obesity on health, continued surveillance of chronic disease and risk factors is warranted, as are further health promotion and health education 
initiatives. We continue to work with the community to develop and evaluate primary and secondary prevention activities.

\section{Acknowledgments}

We thank the Canadian Institutes of Health Research (CIHR) and the Manitoba Health Research Council for their funding for this project. Dr Riediger is the recipient of a CIHR Doctoral Canada Graduate Scholarship. We are grateful for the statistical assistance of Mary Cheang. Finally, we thank the study community, staff, and leadership for their participation and ongoing commitment.

\section{References}

1. Macaulay AC. Improving aboriginal health: how can health care professionals contribute? Can Fam Physician 2009;55(4):334-9.

2. Anand SS, Yusef S, Jacobs R, Davis AD, Yi Q, Gerstein H, et al. Risk factors, atherosclerosis, and cardiovascular disease among Aboriginal people in Canada: the Study of Health Assessment and Risk Evaluation in Aboriginal Peoples (SHARE-AP). Lancet 2001;358(9288):1147-53

3. Allard YE, Wilkins R, Berthelot JM. Premature mortality in health regions with high aboriginal populations. Health Rep 2004;15(1):51-60.

4. Lix L, Bruce S, Sarkar J, Young TK. Risk factors and chronic conditions among Aboriginal and non-Aboriginal populations. Health Rep 2009;20(4):21-9.

5. Connelly PW, Hanley AJ, Harris SB, Hegele RA, Zinman B. Relation of waist circumference and glycemic status to C-reactive protein in the Sandy Lake Oji-Cree. Int J Obes Relat Metab Disord 2003;27(3):347-54.

6. Chateau-Degat ML, Pereg D, Egeland GM, Nieboer E, Bonnier-Viger YV, Laouan-Sidi EA, et al. Diabetes and related metabolic conditions in an aboriginal Cree community of Quebec, Canada. Canadian Journal of Diabetes 2009;33(3):156-62.
7. Oster RT, Toth EL. Differences in the prevalence of diabetes risk-factors among First Nation, Metis and non-aboriginal attending screening clinics in rural Alberta, Canada. Rural Remote Health 2009;9(2):1170.

8. Dyck R, Osgood N, Lin TH, Gao A, Stang MR. Epidemiology of diabetes mellitus among First Nations and non-First Nations adults. CMAJ 2010;182(3):244-56.

9. Gao S, Manns BJ, Culleton BF, Tonelli M, Quan H, Crowshoe L, et al. Prevalence of chronic kidney disease and survival among aboriginal people. J Am Soc Nephrol 2007;18(11):2953-9.

10. Bruce SG, Young TK. Prevalence and risk factors for neuropathy in a Canadian First Nation community. Diabetes Care 2008;31(9):1837-41.

11. Canadian Society for Exercise Physiology. The Canadian Physical Activity, Fitness, and Lifestyle Approach. 3rd edition. Ottawa, Ontario (CA): CSEP; 2003.

12. Canadian Diabetes Association 2008 clinical practice guidelines for the prevention and management of diabetes in Canada. Canadian Journal of Diabetes 2008;32(Suppl 1).

13. Executive Summary of the Third Report of the National Cholesterol Education Program (NCEP) Expert Panel on Detection, Evaluation, and Treatment of High Blood Cholesterol in Adults (Adult Treatment Panel III). JAMA 2001;285(19):2486-97.

14. Armstrong DG, Lavery LA. Clinical care of the diabetic foot. Alexandria (VA): American Diabetes Association; 2005.

15. Garriguet D. Obesity and the eating habits of the aboriginal population. Health Rep 2008;19(1):21-35.

16. Harris SB, Gittelsohn J, Hanley A, Barnie A, Wolever TM, Gao J, et al. The prevalence of NIDDM and associated risk factors in native Canadians. Diabetes Care 1997;20(2):185-7.
17. Aljohani N, Rempel BM, Ludwig S, Morris M, Cheang M, Murray R, et al. Impact of diabetes on maternal-fetal outcomes in Manitoba. Relationship with ethnic and environmental factors. Clin Invest Med 2008;31(6):E338-45.

18. Dunne F, Brydon P, Smith K, Gee H. Pregnancy in women with type 2 diabetes: 12 years outcome data 1990-2002. Diabet Med 2003;20(9):734-8.

19. Young TK, Martens PJ, Taback SP, Sellers EA, Dean HJ, Cheang M, et al. Type 2 diabetes mellitus in children: prenatal and early infancy risk factors among native Canadians. Arch Pediatr Adolesc Med 2002;156(7):651-5.

20. Dyck RF, Klomp H, Tan L. From "thrifty genotype" to "hefty fetal phenotype": the relationship between high birthweight and diabetes in Saskatchewan Registered Indians. Can J Public Health 2001;92(5):340-4.

21. Masharani U, Goldfine ID, Youngren JF Influence of gender on the relationship between insulin sensitivity, adiposity, and plasma lipids in lean nondiabetic subjects. Metabolism 2009;58(11):1602-8.

22. O’Dea K, Cunningham J, Maple-Brown L, Weeramanthri T, Shaw J, Dunbar T, et al. Diabetes and cardiovascular risk factors in urban indigenous adults: results from the DRUID study. Diabetes Res Clin Pract 2008;80(3):483-9.

23. Makaryus AN, Akhrass P, McFarlane SI Treatment of hypertension in metabolic syndrome: implications of recent clinical trials. Curr Diab Rep 2009;9(3):229-37.

24. Amankwah E, Campbell NR, Maxwell C, Onysko J, Quan H. Why some adult Canadians do not have blood pressure measured. J Clin Hypertens (Greenwich) 2007;9(12):944-51. 